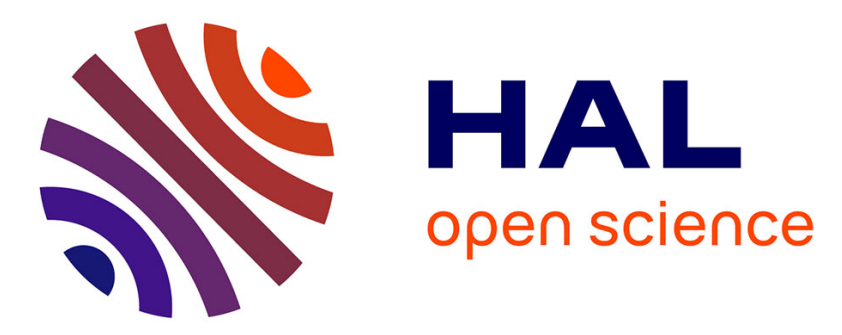

\title{
Constraining Downward Terrestrial Gamma Ray Flashes Using Ground-Based Particle Detector Arrays
}

Nini Berge, Sébastien Celestin

\section{To cite this version:}

Nini Berge, Sébastien Celestin. Constraining Downward Terrestrial Gamma Ray Flashes Using Ground-Based Particle Detector Arrays. Geophysical Research Letters, 2019, 46 (14), pp.8424-8430. 10.1029/2019GL083252 . insu-02263562

\section{HAL Id: insu-02263562 https://hal-insu.archives-ouvertes.fr/insu-02263562}

Submitted on 26 Aug 2019

HAL is a multi-disciplinary open access archive for the deposit and dissemination of scientific research documents, whether they are published or not. The documents may come from teaching and research institutions in France or abroad, or from public or private research centers.
L'archive ouverte pluridisciplinaire HAL, est destinée au dépôt et à la diffusion de documents scientifiques de niveau recherche, publiés ou non, émanant des établissements d'enseignement et de recherche français ou étrangers, des laboratoires publics ou privés. 


\section{Geophysical Research Letters}

\section{RESEARCH LETTER \\ 10.1029/2019GL083252 \\ Constraining Downward Terrestrial Gamma Ray Flashes Using Ground-Based Particle Detector Arrays}

Key Points:

- We model downward directed TGFs in order to be able to better interpret ground observations

- We compare impulse responses at ground and satellite and identify beam geometries on ground

- The number of photons reaching ground is strongly dependent on the initial energy spectrum

Correspondence to:

N. Berge,

nini.berge@cnrs-orleans.fr

Citation:

Berge, N., \& Celestin, S. (2019). Constraining downward terrestrial gamma ray flashes using ground-based particle detector arrays. Geophysical Research Letters, 46, 8424-8430. https://doi.org/10.1029/2019GL083252

Received 12 APR 2019 Accepted 3 JUL 2019

Accepted article online 9 JUL 2019

Published online 16 JUL 2019

C2019. American Geophysical Union. All Rights Reserved.

\author{
N. Berge ${ }^{1(i)}$ and S. Celestin ${ }^{1}$ (iD \\ ${ }^{1}$ LPC2E, University of Orleans, CNRS, Orleans, France
}

\begin{abstract}
Until recently, there were only a few ground-based observations of terrestrial gamma ray flashes (TGFs). Since the Telescope Array in Utah, USA, started reporting detections of high-energy particles correlated with lightning, their number has greatly increased. Ground observations of TGFs represent a valuable addition to space-borne detectors. The proximity to the event and the ability to observe an event with several detectors may reveal new information about the production of TGFs. In this paper, we study downward directed TGFs using Monte Carlo modeling of photon transport through the atmosphere. The Telescope Array-observed pulses of gamma rays spread over periods of a few hundred microseconds. We predict such structures to be observable at satellite altitude, given sufficient time resolution. Additionally, we demonstrate how various source spectra would lead to different number of photons reaching ground, which impacts the conclusions one can draw using observational data.
\end{abstract}

Plain Language Summary Terrestrial gamma ray flashes (TGFs) are bursts of high-energy photons that originate in thunderstorms. TGFs have been routinely observed from space since their discovery. Until recently, there were only a few ground-based observations of TGFs. Their number has increased since the Telescope Array started reporting detections of high-energy particles at the same time as lightning. Ground observations of TGFs represent a valuable addition to detectors in space. They are closer to the source of the event, and it is possible to observe a single event with several detectors. Because of this, ground observations may reveal new information about the production mechanisms of TGFs. We study downward TGFs by modeling photons moving through the atmosphere. The Telescope Array-observed pulses of gamma rays. We predict that similar pulses should be observable by satellites, given sufficient time resolution. TGFs are thought to start out as photons moving in a cone-shaped beam. We want to find the shape of this beam from ground observations, but photons interacting with the atmosphere changes how the beam looks. We determine the beam shape by the photons' positions on ground. We also find that the number of photons reaching ground is dependent on the photons' initial energies.

\section{Introduction}

Terrestrial gamma ray flashes (TGFs) are bursts of high-energy photons of submillisecond duration originating in thunderstorms. TGFs are bright enough to have been routinely observed from space since their discovery by Fishman et al. (1994) using the BATSE instrument aboard the Compton Gamma Ray Observatory. Since then, they have been detected by several other satellites, notably Fermi, the Reuven Ramaty High Energy Solar Spectroscopic Imager (RHESSI), and Astrorivelatore Gamma a Immagini LEggaro (AGILE) (e.g., Briggs et al., 2010; Grefenstette et al., 2009; Marisaldi et al., 2010). The energy spectrum of TGFs suggests that they originate as bremsstrahlung from Relativistic Runaway Electron Avalanches (RREA) (e.g., Dwyer et al., 2012; Gurevich et al., 1992), but the exact initiation mechanism remains disputed (e.g., Dwyer, 2008; Celestin \& Pasko, 2011). TGFs observed from space are considered to be produced between 10 and $15 \mathrm{~km}$ altitude (e.g., Cummer et al., 2014; Xu et al., 2012), in association with the propagation of positive intracloud (IC) lightning (e.g., Cummer et al., 2015).

In recent years, there have also been occasional observations of gamma ray flashes from the ground. Two cases from rocket-triggered lightning (Dwyer et al., 2004; Hare et al., 2016), as well as two cases in association with negative cloud-to-ground (CG) lightning (Dwyer et al., 2012; Tran et al., 2015), have been observed in Florida, USA. Gamma ray flashes have also been observed from ground during Japanese winter thunderstorms (e.g., Enoto et al., 2017; Smith et al., 2018). While the observations by Dwyer et al. (2004), Dwyer et al. (2012), Enoto et al. (2017), and Hare et al. (2016) were made by several detectors simultaneously, the total 
number of photons received was limited, and the events are few and far between. The number of ground observations of gamma ray flashes from thunderstorms increased significantly as unusual observations correlated to lightning activity were reported from the Telescope Array (TA) in Utah, USA (Abbasi et al., 2017, 2018). The TA is a cosmic ray observatory which has an instrument of more than 500 scintillation detectors in a square grid with 1.2-km spacing called the Telescope Array Surface Detector (TASD). The observed events originate less than $5 \mathrm{~km}$ above ground level and are correlated with downward propagation of negative leaders. The large detector grid of the TASD gives an unusual advantage in observing TGFs from the ground-each event is registered across several detectors, giving a much clearer picture of the spatial and temporal shape of the signal than what single observation points are capable of.

To better be able to interpret observations such as those made by the TA, we model downward directed TGFs using a Monte Carlo model of photon transport through the atmosphere. The TGFs are considered to be beamed downward, that is, produced by electrons accelerated in the direction of negative CG lightning. Our aim is to quantify how differences in time structure, geometry, and energy spectrum of the initial gamma ray flash present in ground-based observations. Abbasi et al. (2018) observed time structures consisting of 10- $\mu$ s pulses spread over a few hundred microseconds. We find that such structures should also be observable by satellites, given sufficient time resolution. The detector grid of the TASD gives unprecedented information about the geometry of TGFs. However, atmospheric effects cause distortion of the initial beam shape. To resolve this, we define a parameter space from the observed photons' position, which can uniquely identify most possible geometries of the initial TGF beam. We also find that the number of photons reaching ground, as well as the shape of their energy spectra, strongly depends on the initial spectrum of the TGF.

\section{Model}

We model the behavior of TGFs by simulating individual photons moving through the atmosphere. The model we use is based on the Monte Carlo model described in Østgaard et al. (2008). The photons originate at a point source, and their velocities are distributed uniformly within a cone-shaped beam of predetermined width. Values of the beam width are varied from $10^{\circ}$ to $45^{\circ}$ half-cone angle, which covers the range found in the literature-Abbasi et al. (2018) found their observations to be consistent with a beam with a $16^{\circ}$ half angle and Gjesteland et al. (2011) found a half angle between $30^{\circ}$ and $40^{\circ}$ to be consistent with RHESSI observations. We also used different tilts of the beam, ranging from $0^{\circ}$ to $35^{\circ}$. The source altitude is set as $12 \mathrm{~km}$ for upward TGFs (e.g., Cummer et al., 2014; Xu et al., 2012), and $3 \mathrm{~km}$ for downward TGFs (Abbasi et al., 2017).

Each photon is launched with an initial energy following a predetermined energy spectrum and is given a position and direction from the distribution of a chosen initial geometry. We use the exponential spectrum associated with RREAs with a cutoff energy at 7.3 MeV (e.g., Coleman \& Dwyer, 2006; Dwyer, 2008) as a reference case, as well as spectra associated with different leader potential drops (Celestin et al., 2015).

A path length between particle interactions is determined based on the total probability of interaction. Then, the type of interaction is determined from their relative probabilities given by cross sections. The interactions accounted for are Compton scattering, pair production, and photoelectric absorption. The cross sections for Compton scattering and pair production are taken from the NIST Standard Reference Database of photon cross sections (XCOM; Berger et al., 2010). The cross sections for photoelectric absorption are from Lehtinen (2000). We assume an atmospheric composition of $80 \% \mathrm{~N}_{2}$ and $20 \% \mathrm{O}_{2}$. Each photon is kept track of until its energy falls below $10 \mathrm{keV}$, or until it reaches satellite altitude or the ground. Satellite altitude is set as $500 \mathrm{~km}$ throughout this paper.

\section{Results and Discussion}

\subsection{Time Dispersion}

Figure 1a shows the impulse response of an instantaneously produced TGF on ground and at satellite altitude at radial distance $1,166 \mathrm{~m}$ and $200 \mathrm{~km}$, respectively. Ninety percent of the photons from a simulated downward directed TGF impulse with source at 3-km altitude reach ground in about $1.5 \mu$ s. For an upward TGF impulse originating at $12 \mathrm{~km}$, it spreads over more than $57 \mu \mathrm{s}$. This shows that time dispersion due to scattering of the gamma rays is smaller on the ground than at satellite altitude. We have verified that this is due to shorter distances traveled, not to the number of times each photon is scattered. However, the majority of photons at satellite altitude arrive within a very short time. As an example, the first $60 \%$ arrive 

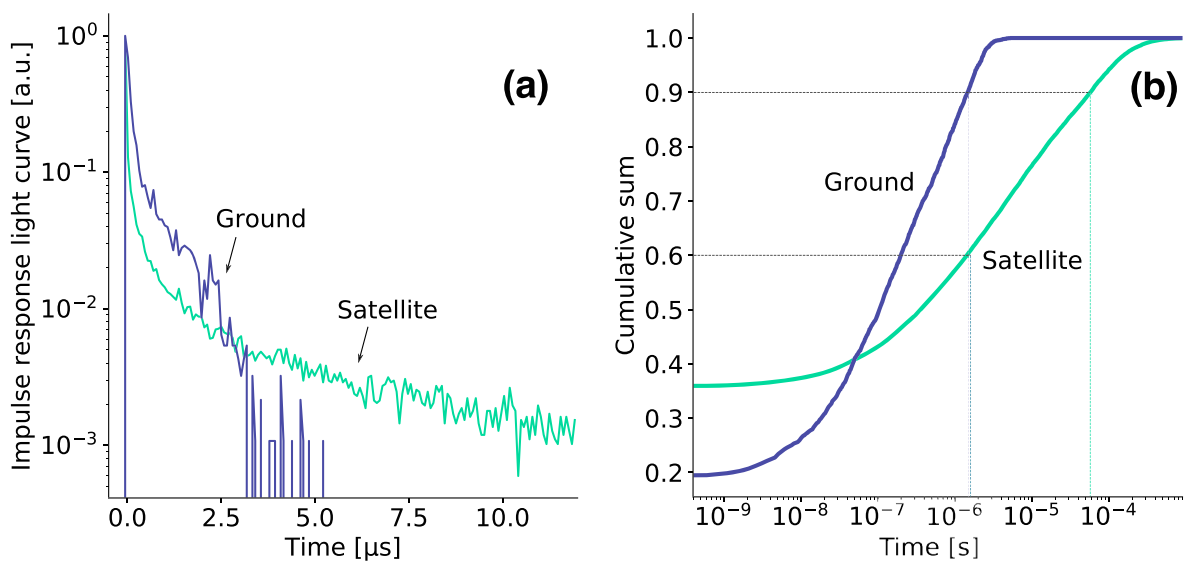

Figure 1. (a) Impulse response of an instantaneous TGF at satellite altitude (green) and ground (blue). Both lines are normalized to peak at 1, to best compare the shape of the light curve. (b) Normalized cumulative sum of photons from an instantaneous TGF arriving at satellite altitude (green) and ground (blue). The points where $60 \%$ and $90 \%$ of photons have arrived are marked with dashed lines.

within $1.6 \mu \mathrm{s}$ (see Figure 1b). The long duration to reach $90 \%$ is due to a minority of photons. Abbasi et al. (2018) observed time structures of less than $10 \mu$ s over a few hundred microseconds. Since the duration of the impulse response is only a few microseconds, we conclude that such structures should be observable at satellite altitude given sufficient time resolution, if they are present in upward TGFs.

Space-based detectors have observed some TGFs to be pulsed. While the duration between pulses is generally in the order of milliseconds (e.g., Fishman et al., 1994; Marisaldi et al., 2014; Mezentsev et al., 2016), overlapping pulses with submillisecond separation also occur (Briggs et al., 2010; Foley et al., 2014; Stanbro et al., 2018). It remains an open question whether this is due to different production mechanisms for the

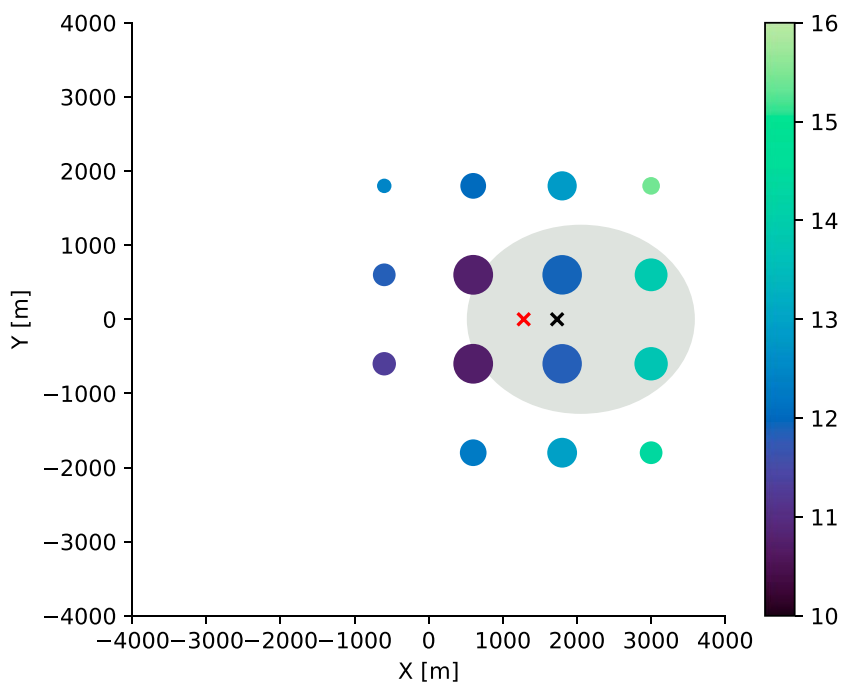

Figure 2. A simulated terrestrial gamma ray flash initiated at origin and 3 -km altitude with a beam width of $20^{\circ}$ and a tilt of $30^{\circ}$, as it would be detected by an ideal detector setup similar to that of Abbasi et al. (2017, 2018). Each point represents one detector. The point size corresponds to the logarithm of the number of photons hitting each detector, while the color corresponds to the median time of arrival in microseconds. The black cross shows where the middle of the projected beam would have hit in vacuum, the ellipse marks the footprint of the beam without atmospheric effects. The red cross marks the mean as calculated from the hits in the detectors. events of Abbasi et al. $(2017,2018)$ and TGFs detected from space, or whether such time structures always exist but are not observable. The latter case could be due to detector limitations.

\subsection{Geometry}

Figure 2 shows simulation results illustrating how an idealized TASD observation could look for a TGF with a tilt of $30^{\circ}$ and a beam width of $20^{\circ}$. It is reminiscent of the detections reported in Abbasi et al. (2017), where the events observed by the TASD also tend to register in more than 10 detectors. This simulation shows the difficulty of accurately inferring the original geometry from such detections. The black cross shows where the middle of the projected beam would have hit in vacuum, and the gray ellipse marks the footprint of the projected beam. The red cross marks the mean as calculated from the hits in the detectors. We can see how the mean is shifted toward the TGF source, here set at origin, as the photons had to traverse less atmosphere on this side of the beam. The observed footprint is also significantly blurred.

To quantify how results of this kind can be used to determine the geometry of the initial beam, we have analyzed a series of simulations with different initial conditions. The beam width was varied from $10^{\circ}$ to $45^{\circ}$, and the beam was tilted between $0^{\circ}$ and $35^{\circ}$ (see Figure 3 for an illustration of the geometry). We define the axis of the tilt, meaning the line between the footpoint of the TGF's initial position and the mean position of the photons that reached ground, as the $x$-direction. These metrics can be applied to real detections, as the source position can be determined using a lightning mapping array (Abbasi et al., 2018), and the mean position is a relatively accurate measure even with discrete detectors. 


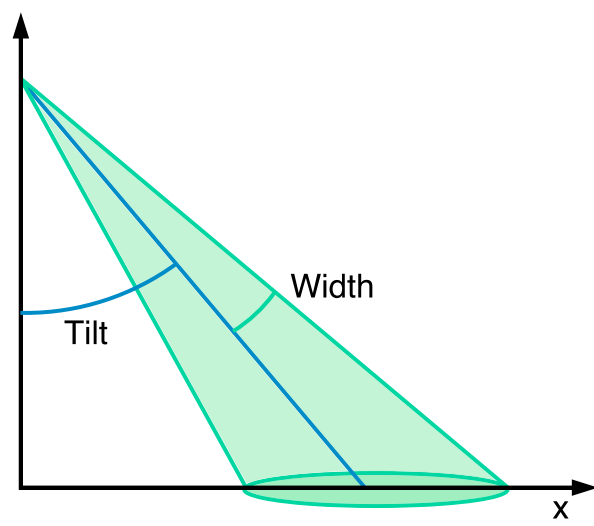

Figure 3. Configuration of the initial beam geometry. The beam width is varied from $10^{\circ}$ to $45^{\circ}$, and the beam is tilted between $0^{\circ}$ and $35^{\circ}$.

Saving the positions of all photons that reached ground in the different configurations, we created a parameter space of the mean and standard deviation of the photons' position along the tilt axis, $x$. The results can be seen in Figure 4, where each point represents a different initial configuration. Most configurations have a uniquely defined position in this parameter space, indicating that the initial geometry can be found. However, a few initial configurations yield similar results and make identification difficult. This concerns combinations of larger tilts $\left(\geq 20^{\circ}\right)$ and very wide beams $\left(>40^{\circ}\right)$. The error bars signify the difference in mean and standard deviation obtained from calculating the values from discrete detectors representative of those used by Abbasi et al. $(2017,2018$ ) (as in Figure 2), rather than using the exact positional values from our simulations. The difference of the mean values is very small, while for the standard deviation the change is larger. The calculated standard deviation will increase with the distance between detectors. The points of the parameter space remain resolved, just shifted upward, for most configurations, especially around the middle of the angular ranges used. This remains the case when the beam is aligned differently with respect to the detector grid. Through simulations, one can calibrate the expected increase in standard deviation to specific detector grids.

To further distinguish detections where this parameter space is inconclusive, one can consider the asymmetry of the detection, that is, how many detections fall below the mean position compared to above. The asymmetry generally increases with both tilt and beam width but is sensitive to the alignment of the beam with the detector grid. Such considerations would therefore have to be made with simulations on a case-to-case basis.

\subsection{Energy Spectra}

The initial energy spectrum of a TGF depends on its exact production mechanism. We use initial spectra corresponding to runaway electrons produced in inhomogeneous fields at leader tips with potential drops from 60 to $300 \mathrm{MV}$ (Celestin et al., 2015) and an exponential spectrum with cutoff at $7.3 \mathrm{MeV}$ as produced by a full RREA developing in a large-scale electric field (e.g., Coleman \& Dwyer, 2006). The different initial spectra can be seen in Figure 5. Different leader potential drops are thought to be associated with different numbers of initial photons, where the harder spectra correspond to a greater number of photons (Celestin et al., 2015), see Table 1. TGFs are generally considered to start out with around $10^{18}$ photons (e.g., Smith et al., 2011; Østgaard et al., 2012). Here we use $2 \cdot 10^{18}$ photons in the RREA case, which is the same number as for the 300-MV potential drop spectrum. We only consider photons with energies greater than $10 \mathrm{keV}$.

Photons lose energy as they propagate through air. Consequently softer spectra have a lower percentage of the photons reaching ground, as more photons will be absorbed during propagation. Table 1 shows the fraction of photons reaching ground for the different initial spectra used. The decrease in number is more severe for softer spectra. The resulting energy spectra on ground with their respective magnitudes can be seen in Figure 5b.

It follows that both the shape of the energy spectrum and the number of photons observed on ground depend on the initial spectrum. This should be taken into account when making estimates of the initial conditions 


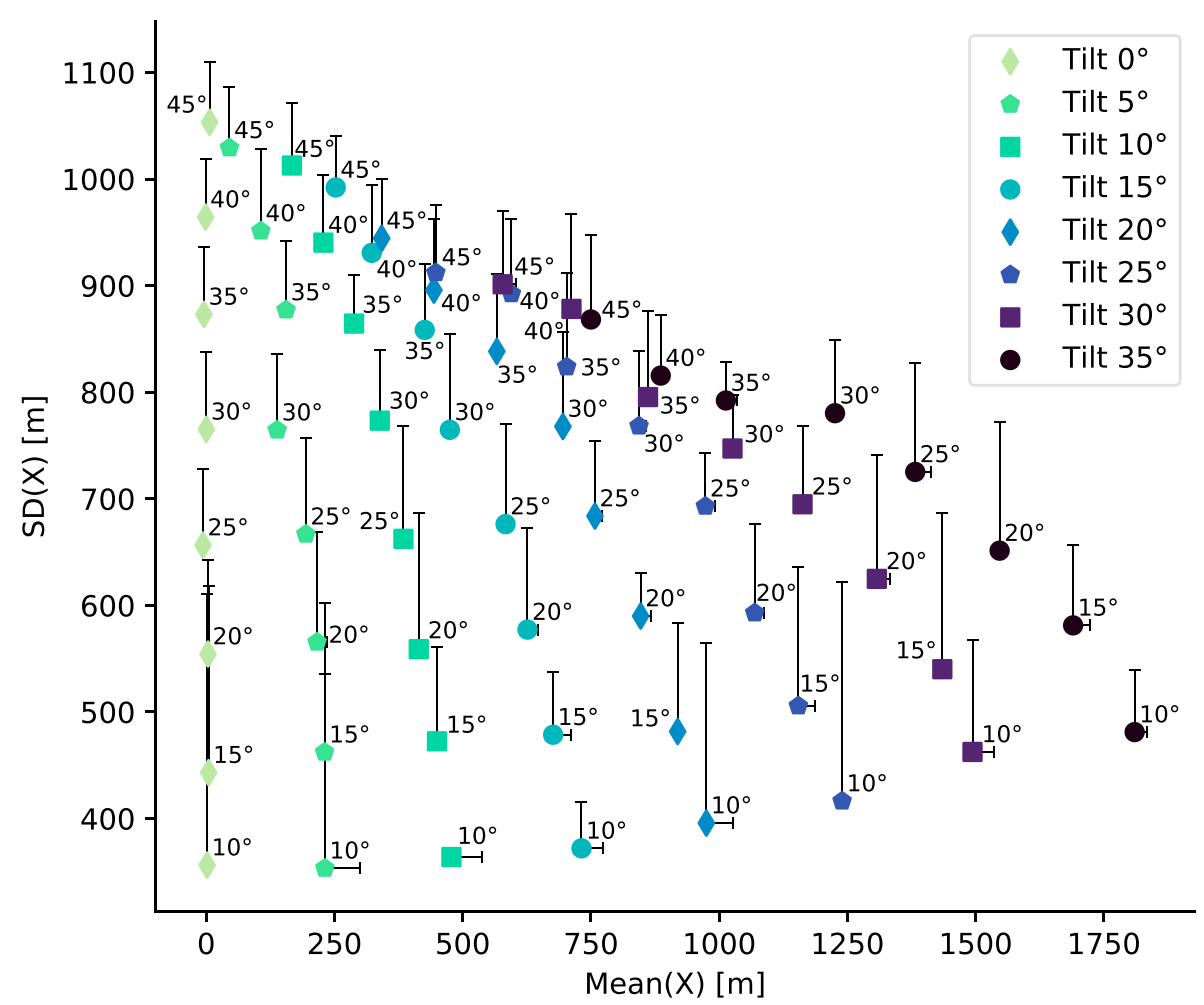

Figure 4. Parameter space with mean position along the $x$ axis versus standard deviation of position along $x$. The $x$ axis is defined as the line between the source and the mean position of detections. Each point on the plot represents one simulation with given initial conditions. The color and shape vary with the tilt angle, while the beam width is printed next to each point. The error bars describe the difference we get if the numbers are calculated from discrete ideal detectors representative of those used by Abbasi et al. $(2017,2018)$, rather than from each photon's exact position.

corresponding to a ground observation, as the difference between the $60 \mathrm{MV}$ and the RREA case is almost an order of magnitude.

As an example, if one considers the case of the $60 \mathrm{MV}$ potential drop and $6 \cdot 10^{14}$ initial photons, only $\sim 4 \cdot 10^{-5}$ of them reach ground, giving a total of $\sim 2 \cdot 10^{10}$ photons that could be observed. For harder spectra, such as the RREA spectrum, the fraction is much higher-out of one, $\sim 4 \cdot 10^{-4}$ reach ground. If one were to have such an observation and calculate the number of initial photons, but using the rate of photons reaching ground with the RREA spectrum, one would find $\sim 6 \cdot 10^{13}$, an order of magnitude too few. As the initial
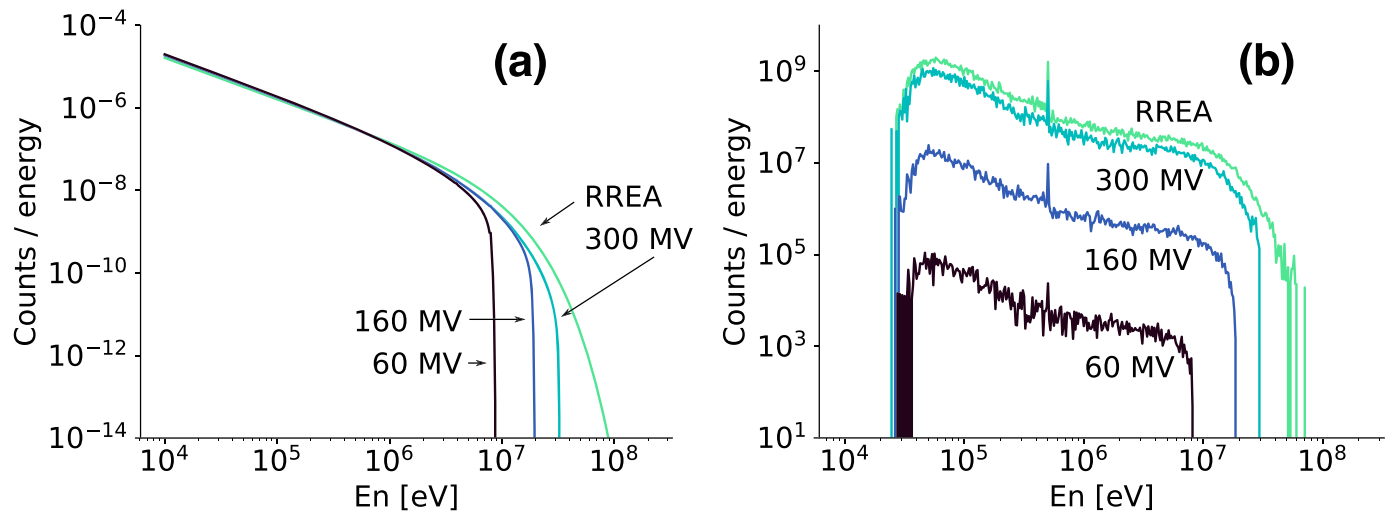

Figure 5. (a) Initial photon energy spectra corresponding to leader potential drops from 60 to $300 \mathrm{MV}$ and a RREA spectrum with cutoff at $7.3 \mathrm{MeV}$. The spectra's magnitudes are aligned, not accounting for a difference in number of photons. (b) Photon energy spectra as they present on ground, corresponding to leader potential drops from 60 to $300 \mathrm{MV}$, and an exponential RREA spectrum with cutoff at $7.3 \mathrm{MeV}$. 
Table 1

Initial Number of Photons and the Simulated Fraction of Photons Reaching Ground for the Different Spectra in Figure 5

\begin{tabular}{lcc}
\hline Spectrum & $\begin{array}{c}\text { Initial number } \\
\text { of photons }\end{array}$ & $\begin{array}{c}\text { Fraction reaching } \\
\text { ground }\end{array}$ \\
\hline $60 \mathrm{MV}$ & $6 \cdot 10^{14}$ & $4.0 \cdot 10^{-5}$ \\
$160 \mathrm{MV}$ & $4 \cdot 10^{16}$ & $1.5 \cdot 10^{-4}$ \\
$300 \mathrm{MV}$ & $2 \cdot 10^{18}$ & $1.9 \cdot 10^{-4}$ \\
RREA & $2 \cdot 10^{18}$ & $3.7 \cdot 10^{-4}$ \\
\hline
\end{tabular}

Note. Initial numbers as function of potential drop are from Celestin et al. (2015).

number of photons associated with softer spectra is presumably lower, this can explain the range of $10^{12}$ to $10^{14}$ initial photons obtained by Abbasi et al. (2018).

\section{Conclusion}

The conclusions of this work can be summarized as follows:

1. Despite the differences in distance traveled by the photons, the time dispersion seen in the impulse responses of TGFs measured at satellite altitude and at ground share a steep peak lasting only microseconds. If pulses of duration shorter than $10 \mu \mathrm{s}$ with tens of microseconds separation occur in the production of TGFs, they should be observable from space, given instrumentation with sufficient time resolution.

2. Most TGF beaming geometries can be uniquely identified by the mean and standard deviation of the detections position along the tilt axis. Further distinction could be done using a measure of the asymmetry along the tilt axis on a case-to-case basis. This demonstrates the great potential of establishing the TGF source position using arrays of evenly spaced ground-based gamma ray detectors along with lightning mapping.

3. The number of photons arriving at the ground is heavily dependent on the photons initial energy spectrum, which may be a source of error when evaluating the number of photons at the source. Abbasi et al. (2018) estimate their observations to correspond to $10^{12}$ to $10^{14}$ initial photons. This could be consistent with a burst of gamma rays produced by lightning leaders forming potential drops of $60 \mathrm{MV}$.

\section{Acknowledgments}

This research was supported by the French space agency (CNES) as part of the TARANIS mission and the Région Centre-Val de Loire. Simulation data allowing to reproduce our figures are available from https://figshare.com/s/ b2272a86bb04c1e876f6.

\section{References}

Abbasi, R. U., Abe, M., Abu-Zayyad, T., Allen, M., Anderson, R., Azuma, R., et al. (2017). The bursts of high energy events observed by the Telescope Array surface detector. Physics Letters A, 381, 2565-2572. https://doi.org/10.1016/j.physleta.2017.06.022

Abbasi, R. U., Abu-Zayyad, T., Allen, M., Barcikowski, E., Belz, J. W., Bergman, D. R., et al. (2018). Gamma ray showers observed at ground level in coincidence with downward lightning leaders. Journal of Geophysical Research: Atmospheres, 123, 6864-6879. https://doi.org/ 10.1029/2017JD027931

Berger, M. J., Hubbell, J. H., Seltzer, S. M., Chang, J., Coursey, J. S., Sukumar, R., et al. (2010). XCOM: Photon cross sections database. https://doi.org/10.18434/T48G6X

Briggs, M. S., Fishman, G. J., Connaughton, V., Bhat, P. N., Paciesas, W. S., Preece, R. D., et al. (2010). First results on terrestrial gamma ray flashes from the Fermi Gamma-ray Burst Monitor. Journal of Geophysical Research, 115, A07323. https://doi.org/10.1029/2009JA015242

Celestin, S., \& Pasko, V. P. (2011). Energy and fluxes of thermal runaway electrons produced by exponential growth of streamers during the stepping of lightning leaders and in transient luminous events. Journal of Geophysical Research, 116, A03315. https://doi.org/10.1029/ 2010JA016260

Celestin, S., Xu, W., \& Pasko, V. P. (2015). Variability in fluence and spectrum of high-energy photon bursts produced by lightning leaders. Journal of Geophysical Research: Space Physics, 120, 10,712-10,723. https://doi.org/10.1002/2015JA021410

Coleman, L. M., \& Dwyer, J. R. (2006). Propagation speed of runaway electron avalanches. Geophysical Research Letters, 33, L11810. https://doi.org/10.1029/2006GL025863

Cummer, S. A., Briggs, M. S., Dwyer, J. R., Xiong, S., Connaughton, V., Fishman, G. J., et al. (2014). The source altitude, electric current, and intrinsic brightness of terrestrial gamma ray flashes. Geophysical Research Letters, 41, 8586-8593. https://doi.org/10.1002/2014GL062196

Cummer, S. A., Lyu, F., Briggs, M. S., Fitzpatrick, G., Roberts, O. J., \& Dwyer, J. R. (2015). Lightning leader altitude progression in terrestrial gamma-ray flashes. Geophysical Research Letters, 42, 7792-7798. https://doi.org/10.1002/2015GL065228

Dwyer, J. R. (2008). Source mechanisms of terrestrial gamma-ray flashes. Journal of Geophysical Research, 113, D10103. https://doi.org/10. 1029/2007JD009248

Dwyer, J. R., Rassoul, H. K., Al-Dayeh, M., Caraway, L., Wright, B., Chrest, A., et al. (2004). A ground level gamma-ray burst observed in association with rocket-triggered lightning. Geophysical Research Letters, 31, L05119. https://doi.org/10.1029/2003GL018771

Dwyer, J. R., Schaal, M. M., Cramer, E., Arabshahi, S., Liu, N., Rassoul, H. K., et al. (2012). Observation of a gamma-ray flash at ground level in association with a cloud-to-ground lightning return stroke. Journal of Geophysical Research, 117, A10303. https://doi.org/10. 1029/2012JA017810 
Dwyer, J. R., Smith, D. M., \& Cummer, S. A. (2012). High-energy atmospheric physics: Terrestrial gamma-ray flashes and related phenomena. Space Science Reviews, 173, 133-196. https://doi.org/10.1007/s11214-012-9894-0

Enoto, T., Wada, Y., Furuta, Y., Nakazawa, K., Yuasa, T., Okuda, K., et al. (2017). Photonuclear reactions triggered by lightning discharge. Nature, 551, 481-484. https://doi.org/10.1038/nature24630

Fishman, G. J., Bhat, P. N., Mallozzi, R., Horack, J. M., Koshut, T., Kouveliotou, C., et al. (1994). Discovery of intense gamma-ray flashes of atmospheric origin. Science, 264, 1313-1316. https://doi.org/10.1126/science.264.5163.1313

Foley, S., Fitzpatrick, G., Briggs, M. S., Connaughton, V., Tierney, D., McBreen, S., et al. (2014). Pulse properties of terrestrial gamma-ray flashes detected by the Fermi Gamma-ray Burst Monitor. Journal of Geophysical Research: Space Physics, 119, 5931-5942. https://doi. org/10.1002/2014JA019805

Gjesteland, T., Østgaard, N., Collier, A. B., Carlson, B. E., Cohen, M. B., \& Lehtinen, N. G. (2011). Confining the angular distribution of terrestrial gamma ray flash emission. Journal of Geophysical Research, 116, A11313. https://doi.org/10.1029/2011JA016716

Grefenstette, B. W., Smith, D. M., Hazelton, B. J., \& Lopez, L. I. (2009). First RHESSI terrestrial gamma ray flash catalog. Journal of Geophysical Research, 114, A02314. https://doi.org/10.1029/2008JA013721

Gurevich, A. V., Milikh, G. M., \& Roussel-Dupre, R. (1992). Runaway electron mechanism of air breakdown and preconditioning during a thunderstorm. Physics Letters A, 165, 463-468. https://doi.org/10.1016/0375-9601(92)90348-P

Hare, B. M., Uman, M. A., Dwyer, J. R., Jordan, D. M., Biggerstaff, M. I., Caicedo, J. A., et al. (2016). Ground-level observation of a terrestrial gamma ray flash initiated by a triggered lightning. Journal of Geophysical Research: Atmospheres, 121, 6511-6533. https://doi.org/10. 1002/2015JD024426

Lehtinen, N. G. (2000). Relativistic runaway electrons above thunderstorms, Stanford University.

Marisaldi, M., Fuschino, F., Labanti, C., Galli, M., Longo, F., Del Monte, E., et al. (2010). Detection of terrestrial gamma ray flashes up to $40 \mathrm{MeV}$ by the AGILE satellite. Journal of Geophysical Research, 115, A00E13. https://doi.org/10.1029/2009JA014502

Marisaldi, M., Fuschino, F., Tavani, M., Dietrich, S., Price, C., Galli, M., et al. (2014). Properties of terrestrial gamma ray flashes detected by AGILE MCAL below 30 MeV. Journal of Geophysical Research: Space Physics, 119, 1337-1355. https://doi.org/10.1002/2013JA019301

Mezentsev, A., Østgaard, N., Gjesteland, T., Albrechtsen, K., Lehtinen, N., Marisaldi, M., et al. (2016). Radio emissions from double RHESSI TGFs. Journal of Geophysical Research: Atmospheres, 121, 8006-8022. https://doi.org/10.1002/2016JD025111

Østgaard, N., Gjesteland, T., Hansen, R. S., Collier, A. B., \& Carlson, B. (2012). The true fluence distribution of terrestrial gamma flashes at satellite altitude. Journal of Geophysical Research, 117, A03327. https://doi.org/10.1029/2011JA017365

Østgaard, N., Gjesteland, T., Stadsnes, J., Connell, P. H., \& Carlson, B. (2008). Production altitude and time delays of the terrestrial gamma flashes: Revisiting the Burst and Transient Source Experiment spectra. Journal of Geophysical Research, 113, A02307. https://doi.org/10. 1029/2007JA012618

Smith, D. M., Bowers, G. S., Kamogawa, M., Wang, D., Ushio, T., Ortberg, J., et al. (2018). Characterizing upward lightning with and without a terrestrial gamma ray flash. Journal of Geophysical Research: Atmospheres, 123, 11,321-11,332. https://doi.org/10.1029/2018JD029105

Smith, D. M., Dwyer, J. R., Hazelton, B. J., Grefenstette, B. W., Martinez-McKinney, G. F. M., Zhang, Z. Y., et al. (2011). The rarity of terrestrial gamma-ray flashes. Geophysical Research Letters, 38, L08807. https://doi.org/10.1029/2011GL046875

Stanbro, M., Briggs, M. S., Roberts, O. J., Cramer, E. S., Cummer, S. A., \& Grove, J. E. (2018). A study of consecutive terrestrial gamma-ray flashes using the Gamma-ray Burst Monitor. Journal of Geophysical Research: Space Physics, 123, 9634-9651. https://doi.org/10.1029/ 2018JA025710

Tran, M. D., Rakov, V. A., Mallick, S., Dwyer, J. R., Nag, A., \& Heckman, S. (2015). A terrestrial gamma-ray flash recorded at the Lightning Observatory in Gainesville, Florida. Journal of Atmospheric and Solar-Terrestrial Physics, 136, 86-93. https://doi.org/10.1016/j.jastp.2015. 10.010

Xu, W., Celestin, S., \& Pasko, V. P. (2012). Source altitudes of terrestrial gamma-ray flashes produced by lightning leaders. Geophysical Research Letters, 39, L08801. https://doi.org/10.1029/2012GL051351 